\title{
Surface sulfation of crab chitin for anisotropic swelling and nanodispersion
}

Xijun Wang, ${ }^{\dagger, \ddagger}$ Pan Chen, ${ }^{*}$, Xiao Feng, ${ }^{\ddagger}$ Chao Dang, ${ }^{\ddagger}$ Baofeng Lin, ${ }^{*, \dagger}$ Yoshiharu Nishiyama, ${ }^{* \S}$ and Haisong Qi*,

$\dagger$ School of Chemistry and Chemical Engineering, Guangxi University, Nanning 530004, China

$\ddagger$ State Key Laboratory of Pulp and Paper Engineering, South China University of Technology, Guangzhou, 510640, China

\Beijing Engineering Research Centre of Cellulose and Its Derivatives, School of Materials Science and Engineering, Beijing Institute of Technology, 100081, Beijing, P.R. China $\S$ Univ. Grenoble Alpes, CNRS, CERMAV, 38000 Grenoble, France

E-mail: panchen@bit.edu.cn; Ibf@gxu.edu.cn; yoshi@cermav.cnrs.fr; qihs@scut.edu.cn

\begin{abstract}
Crab chitin was sulfated under heterogeneous conditions using sulfamic acid in N'Ndimethylformamide to selectively sulfate the microfibril surface. The degree of sulfation followed a first order kinetics assuming a limited available reaction sites on the surface leveling off at bulk degree-of-substitution of 0.4. The reaction rate was proportional to the square of sulfamic acid concentration, suggesting involvement of two sulfamic acid molecules in a reaction. When washed with water after sulfation, the crab shell chitin fragments were anisotropically swollen in the helicoidal direction, revealing regular alternating birefringence under optical microscope. Further mechanical treatment with high-pressure homogenizer lead to slender nanofibrils, whose dimaters were of the
\end{abstract}




\section{- Introduction}

order of $6 \mathrm{~nm}$ according to turbidimetric analysis, in agreement with the Scherrer size estimated from X-ray diffraction line broadening. Both AFM and TEM measurement showed presence further smaller fragments with diameter of 3-4 nm and length of 300 nm. The current approach presents a rapid and efficient modification to chitin, and a strategy for the preparation of stable nanochitins suspension.

Chitin is a linear natural polymer that consists of $\beta$-(1-4)-2-deoxy-2-N-acetamido-D-glucopyranose residues that assembles into semi-crystalline microfibrils inert to most chemicals. It usually forms complex with protein, occurring as structural components and providing mechanical support in various living organisms. Harsh treatments leads to stable colloidal suspension of elongated nanometric particles that self organize into chiral nematic structure similar to cellulose nanocrystals. ${ }^{1-3}$ There is a large panel of potential application as building block of functonal materials common to cellulose nanocrystals or cellulose nanofibers, but in addition, chitin also presents an advantage of being bioresorbable in animal body including human body ${ }^{4}$ which can be an advantage in biomedical applications.

In the beginning, simple hydrolysis using hydrochloric acid has been used to prepare nanochitin, typically at concentration of $2.5 \mathrm{~N}$ with reflux ${ }^{1}$ or $3 \mathrm{~N}$ at $90{ }^{\circ} \mathrm{C},{ }^{5}$ which happens to cleave long fibrils into shorter fragments, as in the case of cellulose. For chitin, this hydrolysis process also remove labile $\mathrm{N}$-acetyl groups on the surface of crystal, while preserving the inside of crystals intact. The surface deacetylation leaves amine groups that are protonated at lower $\mathrm{pH}$, leading to positive surface charge to confer colloidal stability.

One can also selectively deacetylate the crystal surface by alkali treatment. Alkali treatment using highly concentrated sodium hydroxide solutions, typically around $50 \%$, are used to produce water soluble chitosan due to random deacetylation on the chain. However, when sodium hydroxide solution is below $33 \%$, the alkali is not penetrating $\alpha$-chitin, ${ }^{6}$ leaving the crystal interior intact. Thus one can selectively deacetylation the crystalline surface ${ }^{7}$ without 
the acid hydrolysis. The glucosamine residues on the surface can be also selectively cleaved by periodate for further modifications. ${ }^{8}$

Other methods applied to cellulose can also be applied to chitin, namely sulfuric acid hydrolysis ${ }^{9}$ tetramethylpiperidine-1-oxyl (TEMPO) catalyzed oxidation of primary alcohol on the surface. ${ }^{10}$ While N-deacetylation leads to cationic particles, other methods common to nanocellulose preparation leads to introduction of negative charge on the surface. The resulting nanochitin has zwitterionic character where the overall surface potential that depends on the $\mathrm{pH}$ of the medium. Among these conventional methods, the sulfuric acid leads to the introduction of strong acidic group $(\mathrm{pKa}=\sim 2.5)$ but with low density, while TEMPO oxidation leads to a high density of functional groups but of weakly acidic carboxyl groups. In this work, we aim to introduce high density of sulfate half-ester groups on the crystal surface.

Sulfation of cellulose in homogeneous using different sulfation agent is reported, ${ }^{11}$ among which sulfamic acid is the mildest agent. Sulfamic acid sulfation in N'N-dimethylformamide(DMF) has been also used to prepare long cellulose microfibril suspension without mechanical input. ${ }^{12}$ In this work we tried to extend this method to crab-chitin to selectively modify nanochitin surface to modify its macroscopic behavior.

\section{Experimental}

\section{Sample preparation}

Commercially available ground chitin powder from crab shell with typical size of about 20$100 \mu \mathrm{m}$ were used. (macroscopic density: $0.36 \mathrm{~g} / \mathrm{cm}^{3}$ ) The sample was further sequentially purified using sodium hydroxide solution, hydrochloric acid and hydrogen peroxide and freeze dried. The typical sulfation medium for $1 \mathrm{~g}$ of chitin powder is $2.5 \mathrm{~g}$ of sulfamic acid dissolved in $50 \mathrm{~mL} \mathrm{DMF}$, heated at $80^{\circ} \mathrm{C}$ in an oil bath typically for 5 minutes. We also varied the quantity of sulfamic acid, temperature and duration to study the reaction kinetics. 
The reaction was terminated by vacuum filtration on a PTFE membrane (pore size $=$ $0.2 \mu \mathrm{m}$ ) followed by washing with DMF by repeated resuspension and filtration to wash out the excess sulfamic acid. Typically the operation was repeated 5 times with a 3 -fold dilution with DMF. At each cycle we waited 10 minutes for the diffusion of unreacted sulfamic acid and side products from the powder. The filtrate remained a well-draining compact powder at this stage. The sample was then further washed by water. This was done by repeatedly suspending in deionized water followed by centrifugation at $7000 \mathrm{G}$ for 10 minutes since the sample kept high amount of water was impossible to drain through filter. Three-fold dilution was repeated about 15 times until the $\mathrm{pH}$ of the supernatant remained neutral.

The water swollen powder was further diluted to $0.2 \%$ and dispersed by using highpressure homogenizer passing through $200 \mu \mathrm{m}$ zirconia nozzle at $80 \mathrm{MPa}$. This homogenization lead to a translucent suspension that looks bluish under reflecting light. A scheme illustrating the preparation process is shown in Figure S1.

\section{Characterisation}

Optical microscopy Powder particles were observed using polarizing microscope BX53M (Olympus, Japan), under crossed-polarizer and with insertion of retardation plate.

Elemental analysis Carbon, hydrogen, nitrogen and sulfur contents were measured using elemental analyzer Vario EL cube (Elementar, Germany). The singal intensity was calibrated by using sulfanilamide to obtain the element weight fraction. Taking into account the oxygen in chitin and sulfated chitin, the sum of the weight fractions were 100\% within $5 \%$ error.

Conductimetric titration $20 \mathrm{~mL}$ of $0.2 \%$ chitin suspension was titrated with $10 \mathrm{mM}$ $\mathrm{NaOH}$, by step-wising adding $0.1 \mathrm{~mL}$ using pipette. The total amount of $\mathrm{NaOH}$ added to achieve the minimum conductivity was used as equivalent of sulfate group in the system to deduce the degree of sulfation, assuming that all sulfate group s are in acid form in the beginning . 
Infrared spectroscopy Freeze-dried powder was mixed to about 100:1 ratio with $\mathrm{KBr}$, and finely ground to powder with mortar and pestle,

X-ray diffraction X-ray diffractometer (Bruker D8, Advance, Germany) with a Cu tube in line focus mode was operated at $40 \mathrm{kV}$ and $30 \mathrm{~mA}$ in symmetric reflection geometry, and scanned with a scanning rate of $10^{\circ} / \mathrm{min}$.

Electron microscopy Nanofibers suspensions diluted to about $0.01 \%$ was deposited onto a carbon membrane on a copper grid. Then $2 \%$ uranyl acetate drop was added, and waited for 90 seconds before removing the excess liquid by filtration paper tip. The sample was then dried overnight at room temperature, and then observed by using a transmission electron microscope (JEM-1400plus, Japan), operating at $90 \mathrm{kV}$.

Atomic force microscopy Nanofibers suspensions diluted to about $0.01 \%$ was deposited onto a freshly cleaved mica and dried at ambient temperature overnight. Observation was made using Dimension Icon (Bruker Billerica MA, Germany) with a tapping mode.

\section{Results and discussion}

\section{Anisotropic swelling in water}

The initial apparent density of the starting chitin powder corresponds to a porosity of about $75 \%$ assuming the crystal density of $1.46 \mathrm{~g} / \mathrm{cm}^{313}$ before treatment and DMF hardly increased the apparent volume. On the other hand water was extensively swelling the structure. The swollen sulfated chitin powder was holding 16 to 25 times its dry weight even after centrifugation at $7000 \mathrm{G}$ for 10 minutes. Contrary to the prolonged sulfation for algal cell wall, ${ }^{12}$ chitin powder mostly kept its integrity, though at higher sulfation, part of the sample was lost probably because they detached as isoletad fibrils from the fragment. The 

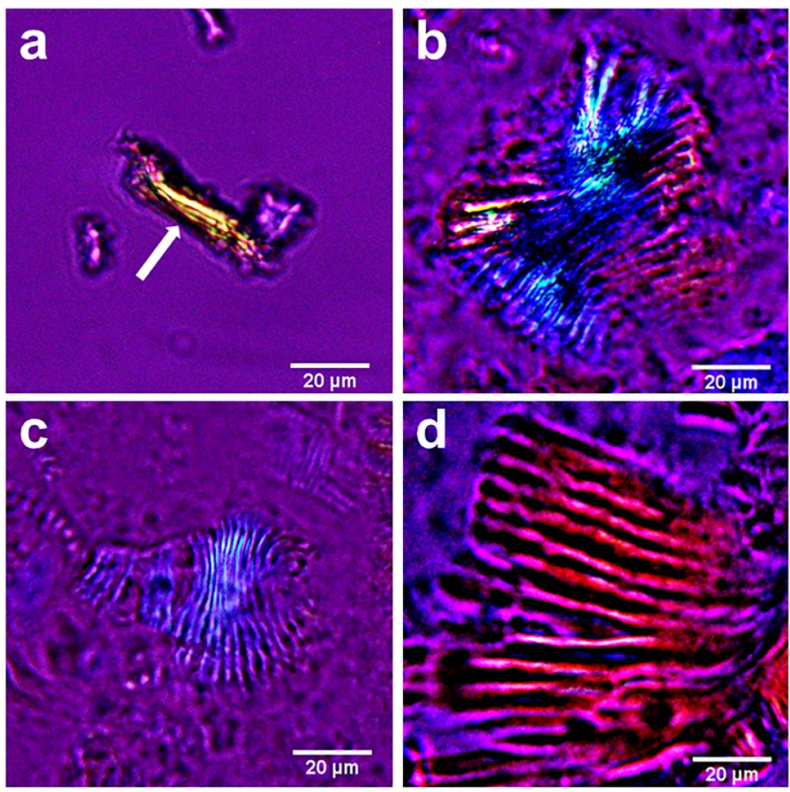

Figure 1: Optical micrographs of water swollen chitin powders under crossed polarizers with a retardation plate. a. pristine chitin powders, b. after 5 min sulfation, c after 15 min sulfation and d after 30 min sulfation.

1 recovery of the centrifugation thus dropped to about $60 \%$ when the sulfation reaction was 2 carried out for more than 1 hour in this conditions.

3

Figure 1 and S2 show the optical micrographs of chitin powder in water before and after sulfation process. Most of the unmodified chitin powder particles showed little birefringence probably because the relatively flat flakes where fragmennts were parallel to the exo-skeleton plane. The chitin microfibrils (and thus the chains) are running in all directions due to the helical structure. Only fragments that happened to be upright show strong birefringence (Figure 1a arrow head). When sulfated powder is swollen in water, the particles show periodic stripes of birefringent region that shows up bright under crossed-polarizers at $45^{\circ}$ with repect to the polarizing direction(Figure S2). Insertion of a retardation plate of $530 \mathrm{~nm}$ shows the yellow and blue color of the stripes depending on the orientation of the stripe. This is due to the birefringence of chitin ${ }^{14}$ and the helicoidal structure of crab exoskeletons. ${ }^{15,16}$ Dark zone correponds to the region where the chitin chains points to the direction of observation.

The fact that we observe more fragments showing this stripe pattern in the highly swollen particles after sulfation suggests a highly anisotropic swelling inverting the aspect ratio of the 
1 particle, probably transforming the oblate fragments into prolate form where the long axis

2 is now in the helical direction, thus lay parallel to the glass slide. We observe a typical half pitch of 4-5 $\mathrm{mm}$. The chitral half-pitch of crab exoskeleton is reported to be predominantly 4 slightly smaller than $1 \mu \mathrm{m}^{16}$ and thus it would suggest that the swelling along the helical axe 5 is around 5 times. As the volume swelling was 16-25 times, the cross-sectional area would 6 have swellen by $4-5$ times. This corresponds to 2-2.5 times expansion in each direction. This 7 situation happens if the interfibrillar distances are multiplied by 5 in a helicoidal organization.

$8 \quad$ How the structure is still maintaining the integritiy in this highly swollen situation is not clear, but since they are only loosely held together a simple mechanical homogenization completely disrupt the integrity as seen below.

\section{Nanochitin dispersion}

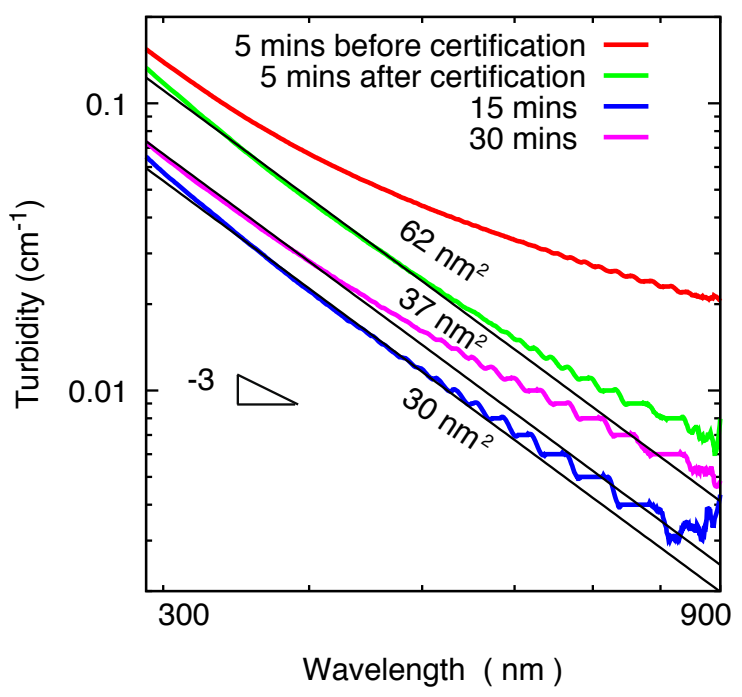

Figure 2: Turbidity of chitin suspensions as function of wavelength. The minutes on the legend indicates the duration of sulfation reaction at $80^{\circ} \mathrm{C}$. Straight lines are theoretical lines for infinite long fibrils of indicated the cross-sectional area.

The homogenized suspension was slightly bluish in reflection and yellow-reddish in transmission by naked eyes indicative of fine scattering elements with dimensions smaller than 



Figure 3: Micrographs of nanochitin after $5 \mathrm{~min}$ sulfation at $80{ }^{\circ} \mathrm{C}$. a) AFM image of nanochitin deposited on mica surface, with insert height profile along the red line in the image. b) Negative stain transmission electron micrographs with width histogram in the insert. 

showed similar morphology shown in Figure S3.

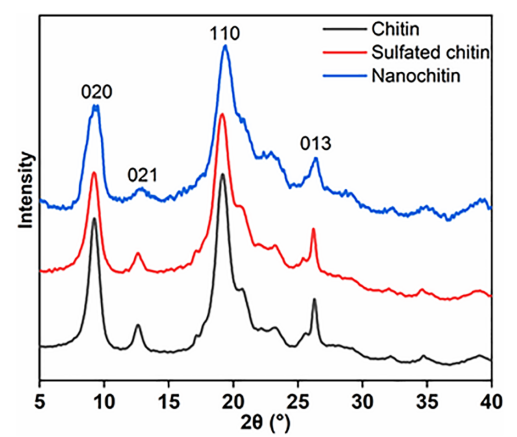

Figure 4: X-ray diffraction profiles of chitin and nanochitin. Sulfation was done at $80^{\circ} \mathrm{C}$ using $50 \mathrm{gL}^{-1}$ sulfamic acid for $5 \mathrm{~min}$.

When further diluted in water and deposited on freshly cleaved mica or on carbon grid, we observed typical height of the order of 3 - $4 \mathrm{~nm}$ by AFM and width of $3-5 \mathrm{~nm}$ by negative staining transmission electron microscopy, while the lengths of straight segments were a few hundred nanometers. The straight segments seem to be linked through kink to make a fibril with contour length of $0.5-1 \mu \mathrm{m}$ length. Nanochitin after 15 and 30 mintues treatment

(1)

(1)

The X-ray diffraction after sulfation and homogenization (Figure 4) showed the same crystalline structure but with broader line width indicating smaller crystallite width. Sulfation alone broadened the line slightly, and the homogenization enhanced this tendency(Figure 4, Figure S2). Since there is no loss in the amount of sample, this decrease in size should be the result of splitting. The Scherrer size based on 020 reflection decreased form $8.2 \mathrm{~nm}$ to $6.5 \mathrm{~nm}$ by sulfation, and further down to $5.5 \mathrm{~nm}$ by the following homogenization (table S1). Still this size is significantly larger than the particles observed under TEM or AFM (3-4 $\mathrm{nm})$. THis is because X-ray diffraction and turbidity represents the volume average of the lateral size, and the larger particles, though small in number, is representing a larger volume in the system. 


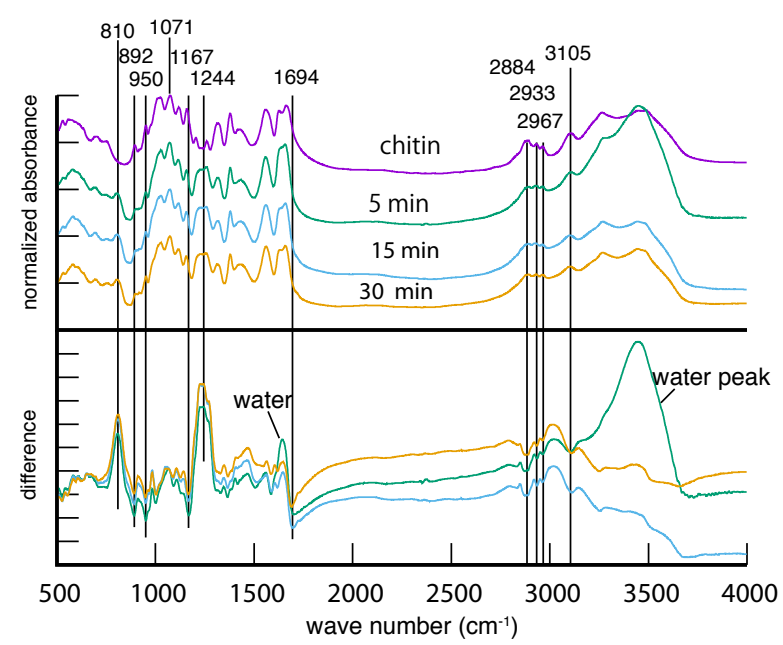

Figure 5: Infrared spectra of chitin and sulfated nanochitin. Top: normalized to the absorbance at $1071 \mathrm{~cm}^{-1}$ and shifted for clarity. Bottom: difference spectra subtracting the unreacted chitin spectra from sulfated chitin. The time on the spectra indicates the duration of sulfation reaction at $80^{\circ} \mathrm{C}$ using $50 \mathrm{gL}^{-1}$ sulfamic acid.

\section{Infrared spectra and site of esterification}

2 Infrared spectra of chitin treated with excess sulfamic acid with concentration $50 \mathrm{~g} / \mathrm{L}$ in

$\mathrm{DMF}$ at $80^{\circ} \mathrm{C}$ as function of duration of reaction is presented in Figure 5. Their spectroscopic features are similar to untreated chitin, except the new bands appearing at $804 \mathrm{~cm}^{-1}$ and $1240 \mathrm{~cm}^{-1}$ characteristic peak to sulfate half-ester groups. In cellulose sodium sulfate, the corresponding peaks appear at 814 and $1260 \mathrm{~cm}^{-1} .{ }^{19}$ When taking difference spectra with respect to the pristine $\alpha$-chitin, we can also recognize the reduction of $892 \mathrm{~cm}^{-1}$ probably corresponding to the surface primary alcohol of the microfibril surface. In cellulose similar band exists at $897 \mathrm{~cm}^{-1}$ that is much less intense in thick microfibrils ${ }^{20}$ and is also negatively correlated to crystallinity. ${ }^{21}$ Clear negative peak can be seen on the higher wavelength limit of the amide I band at $1694 \mathrm{~cm}^{-1}$. The position of amide I band is highly correlated to hydrogen bonding, with isolated amide showing peaks at higher wavenumbers. ${ }^{22}$ This indicates that the surface acetamide would have been removed. Also the $\mathrm{CH}$ stretching peaks at around $2884 \mathrm{~cm}^{-1}$ are decreased after sulfation, which further supports that deacetylation is also occuring in the process which might be due to transesterification at the acetamide moiety. 
1 Thus both O6 and N2 of the surface can be sulfated. Potentially O3 can be also sulfated,

2 but the kinetic studies on cellulose indicates very low activitiy of O3 towards sulfation. ${ }^{11,23}$

\section{Degree of sulfation}

4 Although the general infrared features were consistent among different samples, the sulfate

peak intensity did not follow the reaction severity in the current measurement condition, and thus could not be used to quantify the degree of sulfation.

The sulfate content can be estimated from elemental composition under different assumptions, using sulfur to carbon ratio, $r_{S / C}$ or sulfur to nitrogen ratio, $r_{S / N}$. One can consider two extreme cases: (1) N-acetyl groups are not participating and thus the number of carbon remains constant, so he degree of sulfation is simply

$$
D S_{1}=8 r_{S / C}
$$

(2) only N-acetyl groups are reacting, thus each sulfation reaction removes two carbon atoms and adds one sulfur atom, leading to

$$
D S_{2}=\frac{8 r_{S / C}}{1+2 r_{S / C}} .
$$

Also, if we consider that the number of $\mathrm{N}$ is not changing $D S_{3}=r_{S / N}$.

Conductimetric titration is another way to estimate the degree of sulfation. By neutralizing the highly mobile counter-ion, $\mathrm{H}^{+}$, with less mobile sodium ion, the conductivity of the suspension decrease until complete neutralization. Passed this neutralization, the conductivity increase again due to the highly mobile $\mathrm{OH}^{-}$. The DS obtained by elemental analysis were plotted against DS against conductimetric titration in Figure 6 showing linear correlation but with the conductimetric DS systematically lower than the DS estimated from the elemental analysis by more than factor 2 . One reason for the discrepancy can be the existence of ammonium as counter-ion that contribute little to the conductivity. The presence 


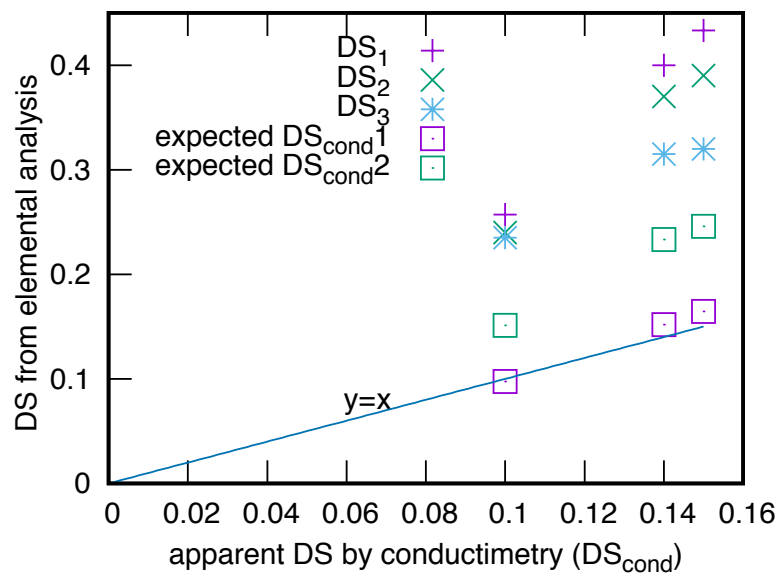

Figure 6: Degree of sulfation estimated by elemental analysis and conductimetric titration

1 of ammonium is also evidenced by the fact that the $\mathrm{DS}_{3}$ estimated from sulfer to nitrogen 2 ratio is systematically lower than the DS estimated from $\mathrm{S} / \mathrm{C}$ ratios. Since we do not have 3 information on the regio-selectivity of the surface sulfation on chitin, we consider the two 4 extreme cases $\mathrm{DS}_{1}$ and $\mathrm{DS}_{2}$. The part, $r$, of the sulfation with ammonium counterion can 5 be estimated as

$$
r=\left(D S_{i}-D S_{3}\right) / D S_{i} D S_{3}
$$

6 which were 0.6 for $\mathrm{DS}_{1}$ and 0.4 for $\mathrm{DS}_{2}$. Given a DS, the conductimetric titration should 7 measure the remaining sites as

$$
D S_{\text {cond }}=(1-r) D S_{i}
$$

8 This expected titration taking into account the presence of ammonium as counterion is 9 plotted as expected $\mathrm{DS}_{\text {cond }}$ in Figure 6. The scenario 1 is closet to the measured values, and is consistent to the general observation that $\mathrm{O} 6$ would be most susceptible to sulfation. On the other hand, DS exceeding 0.4 is difficult to conceive if one considers the typical crystal size of above $5 \mathrm{~nm}$ as estimated by X-ray line broadening, and only surface chains can be sulfated every two residues. Since infrared spectra also suggested some transesterification, 
1 it is still not clear if primary alcohol is predominantly modified. Further analysis is required

2 on the regio-selectivity of sulfation. We tentatively take the $\mathrm{DS}_{1}$ as the degree of sulfation 3 in the following discussion.

\section{Kinetics of sulfation}

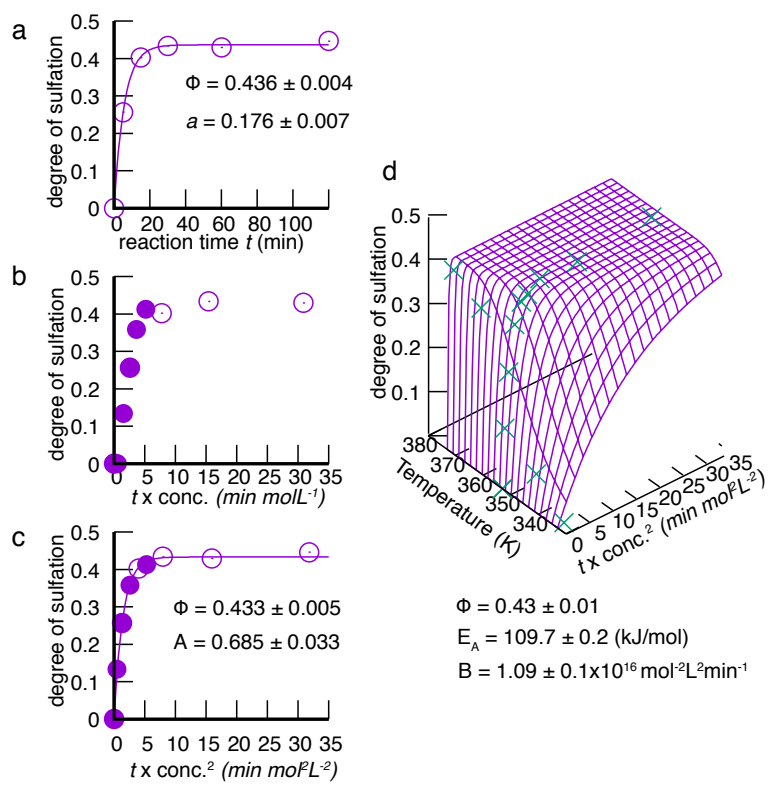

Figure 7: Kinetics of sulfation. Degree of sulfation as function a) reaction time with fixed sulfamic acid concentration of $0.5 \mathrm{molL}^{-1}$ and temperature at $80{ }^{\circ} \mathrm{C}$. b) product of concentration and reaction time; filled circles are series with variable concentration. c) product of concentration squared and reaction time, d) as function of both absolute temperature and $c^{2} t$ with fit to a simple kinetic model (see text).

If we consider that a limited fraction of reactive groups are exposed to the sulfation 6 reagent, and the sulfation reagent is in large excess, the reaction should follow first order7 kinetics with respect to the available reactive groups, and degree of substitution will be 8 expressed as

$$
D S=\phi(1-\exp (-a t))
$$

9 where $\phi$ is the fraction of accessible N-acetyl group to be reacted (considering only transesterification to occur), $a\left[\mathrm{~min}^{-1}\right]$ the kinetic constant and $t$ [min] the duration of reaction. 
1 The DS calculated from elemental analysis indeed followed this curve (Figure 7a). As the

2 frequence of a molecule of sulfamic acid to encounter the reactive groups would scale with the 3 concentration of sulfamic acid, one would expect $a$ to be proportional to the concentration $4 \quad c\left[\mathrm{molL}^{-1}\right]$, and thus

$$
D S=\phi(1-\exp (-b c t))
$$

5 with a kinetic constant $b\left[\mathrm{~mol}^{-1} \mathrm{Lmin}^{-1}\right]$. However, when we plot the DS including series with 6 different sulfamic acid concentration, the curve does not superpose (Figure 7b. Instead, if 7 we plot DS against $c^{2} t$, all experimental data reacted $80^{\circ} \mathrm{C}$ aligned on one line (Figure $7 \mathrm{c}$. 8 This implies that probably two molecules of sulfamic acid is involved in one reaction. The experimental data could be fitted using the following function.

$$
D S=\phi\left(1-\exp \left(-A c^{2} t\right)\right)
$$

Finally we fitted the DS as function of temperature $T[\mathrm{~K}], t$ and $c$ assuming there is one dominant activation energy $E_{A}$

$$
D S=\phi\left(1-\exp \left(-B c^{2} t \exp \left(\frac{-E_{A}}{R T}\right)\right)\right)
$$

where $\mathrm{R}$ is the gas constant $\left(8.3 \mathrm{JK}^{-1} \mathrm{~mol}^{-1}\right)$. The energy of activation $E_{A}$, the prefactor $\mathrm{B}$ and $\phi$ were fitted and shown in Figure $7 \mathrm{~d}$. The activation energy of $109 \mathrm{~kJ} / \mathrm{mol}$ is within the range of typical acetylation reactions of cellulose, which is reported to be between 40-70 $\mathrm{kJ} / \mathrm{mol}$ in dissolving acetylation process, ${ }^{24}$ and $130 \mathrm{~kJ} / \mathrm{mol}$ for heterogeneous acetylation of wood meal. ${ }^{25}$

\section{Conclusion}

We demonstrated that heterogeneous sulfation, selectively sulfating the microfibril surface of crab-chitin, can be achieved using sulfamic acid as sulfating agent in a polar aprotic solvent 


\section{, Acknowledgement}

DMF. The sulfated chitin maintained its integrity up to a bulk DS of 0.4 when gently handled, but could be dispersed in to nanofibrils by high-pressure homogenization. The kinetics of sulfation followed first-order to the available reaction site, and second order to the sulfamic acid concentration suggesting a catalytic role of sulfamic acid in addition to be the reactant. This approach allows a well controlled selective sulfation for the preparation of nano-chitin elements.

We are indebted to staffs of Analytical and Testing Center of SCUT: Dr. Andi Wang for XRD measurement, Dr Qingyou Liang and Dr. Shenghui Zeng for infrared spectroscopy. We also thank colleagues from SCUT/State key laboratory of Pulp and Paper engineering: Dr. Weiying Li for elemental analysis, Dr Yanbo Huang for the assistance in AFM observations and Shanyong Wan for help in polarized optical microscopy. Dr. Jean-Luc Putaux of CERMAV, France for the preliminary TEM observation of sulfated chitin nanoparticles and NanoBio-ICMG Platform (UAR 2607, Grenoble) for granting access to the Electron Microscopy facility. The transmission electron electron microscopy in this article was carried out by Shiyanjia Lab (www.shiyanjia.com). The work was financially supported by the National Natural Science Foundation of China (21774036), Guangdong Province Science Foundation (2017GC010429) and Science and Technology Program of Guangzhou (202002030329).

\section{Supporting Information Available}

Schematic illustration of sample preparation, calculation detail of the cross-section calculation from the turbidimetry data, polarized optical micrographs without retardation plate, atomic force micrographs of nanochitin sulfated for $15 \mathrm{~min}$ and $30 \mathrm{~min}$ at $80^{\circ} \mathrm{C}$, X-rau diffraction profiles of sulfated chitin, nanochitin and its corresponding Scherrer size, primary data of elemental analysis are provided in the supporting information file. 


\section{References}

2 (1) Marchessault, R. H.; Morehead, P. P.; Walter, N. M. Liquid crystal system from Fibrillar

3 Polysaccharides. Nature 1959, 184, 632-633.

(2) Revol, J. F.; Marchessault, R. H. In vitro chiral nematic ordering of chitin crystallites. Int. J. Biol. Macromol. 1993, 15, 329-335.

(3) Belamie, E.; Davidson, P.; Giraud-Guille, M. M. Structure and Chirality of the Nematic Phase in $\alpha$-Chitin Suspensions. J. Phys. Chem. B 2004, 108, 14991-15000.

(4) Escott, G. M.; Adams, D. J. Chitinase Activity in Human Serum and Leukocytes. Infection and Immunity 1995, 65, 4770-4773.

(5) Goodrich, J. D.; Winter, W. T. $\alpha$-Chitin Nanocrystals Prepared from Shrinp Shell and Their Specific Surface Area Measurement. Biomacromolecules 2007, 8, 252-257.

(6) Thor, C. H. B.; Henderson, W. F. Alkali Chitin. American Dyestuff Reporter 1940, 29 , 461-464.

(7) Fan, Y.; Saito, T.; Isogai, A. Individual chitin nano-whiskers prepared from partially deacetylated $\alpha$-chitin by fibril surface cationization. Carbohyd. Polym. 2010, 79, 10461051.

(8) Liu, P.; Liu, H.; Schäfer, T.; Gutman, T.; Gibhardt, H.; Qi, H.; Tian, L.; Zhang, X. C.; Buntkowsky, G.; Zhang, K. Unexpected selective alkaline periodate oxidation of chitin for the isolation of chitin nanocrystals. Green Chemistry 2021, 23, 745-751.

(9) Huang, Y.; Yao, M.; Zheng, X.; Liang, X.; Su, X.; Zhang, Y.; Lu, A.; ; Zhang, L. Effects of Chitin Whiskers on Physical Properties and Osteoblast Culture of Alginate Based Nanocomposite Hydrogels. Biomacromolecules 2015, 16, 3499-1507.

(10) Fan, Y.; Saito, T.; Isogai, A. Chitin Nanocrystals Prepared by TEMPO-Mediated Oxidation of $\alpha$-Chitin. Biomacromolecules 2008, 9, 192-198. 
1 (11) Wagenknecht, W.; Nehls, I.; Köts, J.; Philiipp, B.; Ludwig, J. Untersuchungen zur sulfatierung teilsubstituierter celluloseacetate unter homogenen reaktions bedingungen. Cellul. Chem. Technol. 1991, 25, 343-354.

(12) Briois, B.; Saito, T.; Pétrier, C.; Putaux, J.-L.; Nishiyama, Y.; Heux, L.; MolinaBoisseau, S. $\mathrm{I}_{\alpha} \rightarrow I_{\beta}$ transition of cellulose under ultrasonic radiation. Cellulose 2013, 20, 597-603.

(13) Minke, R.; Blackwell, J. The structure of $\alpha$-Chitin. J. Mol. Biol 1978, 120, 167-181.

(14) Castle, E. S. The double refraction of chitin. J Gen. Physiol. 1936, 19, 797-805.

(15) Bouligand, Y. Sur une architecture torsadée Répandue dans de nombreuses cuticules d'Arthropodes. Compte Rendues Hebtomadaire des séances de l'Académie des sciences 1965, 261, 3665-3668.

(16) Giraud-Guille, M.-M. Fine structure of the chitin-protein system in the crab cuticle. Tissue \&f Cell 1984, 16, 75-92.

(17) Tanaka, R.; Kuribayashi, T.; Ogawa, Y.; Saito, T.; Isogai, A.; Nishiyama, Y. Ensemble evaluation of polyisperse nanocellulose dimensions: rheology, electron microscopy, Xray scattering and turbidimetry. Cellulose 2017, 24, 3231-3242.

(18) Carr Jr., M. E.; Hermans, J. Size and Density of Fibrin Fibers from Turbidity. Macromolecules 1978, 11, 46-50.

(19) Chen, G.; Zhang, B.; Zhao, J.; Chen, H. Improved process for the production of cellulose sulfate using sulfuric acid/ethanol solution. Carbohyd. Polym. 2013, 95, 332-337.

(20) Åkerholm, M.; Hinterstoisser, B.; Salmén, L. Characterization of the crystalline structure of cellulose using static and dynamic FT-IR Spectroscopy. Carbohyd. Res. 2004, 339, 569-578. 
1 (21) Nelson, M. L.; O'Connor, R. T. Relation of certain infrared bands to cellulose crys2 tallinity and crystal lattice type. Part I. spectra of lattice types I, II, III and of amor$3 \quad$ phous cellulose. J Appl. Poly. Sci. 1964, 8, 1311-1324.

4 (22) Torii, H.; Tatsumi, T.; Kanazawa, T.; Tasumi, M. Effect of Intermolecular Hydrogen5 Bonding Interactions on the Amide I Mode of N-Methylacetamide: Matrix-Isolation $6 \quad$ Infrared Studies and ab Initio Molecular Orbital Calculations. J. Phys. Chem. B 1998, $7 \quad$ 102, 309-314.

8 (23) Wagenknecht, W.; Nehls, I.; Philipp, B. Studies on the regioselectivity of cellulose $9 \quad$ sulfation in an $\mathrm{N}_{2} \mathrm{O}_{2}-\mathrm{N}, \mathrm{N}-$ dimethylformamide-cellulose system. Carbohyd. Res 1993, $240,245-252$.

(24) Luo, P.; Cao, C.; Liang, Y.; Ma, X.; Xin, C.; Jiao, Z.; Cao, J.; Zhang, J. Kinetic Study of the Acetylation of Cotton Linter Pulp. BioResources 2013, 8, 2708-2718.

(25) Minato, K.; Ito, Y. Analysis of the factors influencing the acetylation rate of wood. $J$ Wood Sci. 2004, 50, 519-523. 


\section{- TOC Graphic}

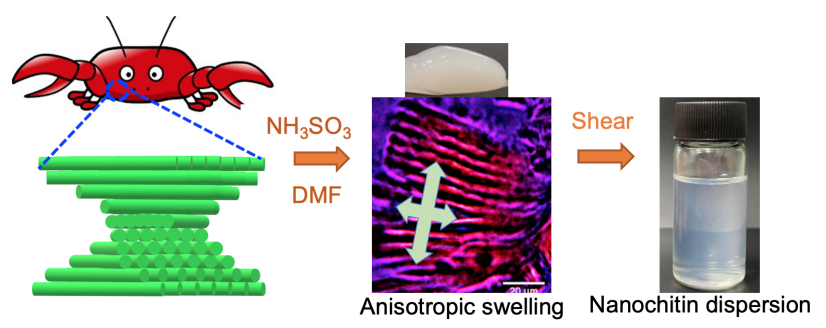

